\title{
Do Near-Solar-System Supernovae Enhance Volcanic Activities on Earth and Neighbouring Planets on Their Paths through the Spiral Arms of the Milky Way, and What Might Be the Consequences for Estimations of Earth's History and Predictions for Its Future?
}

\author{
Heinz-Juergen Brink \\ Institute of Geophysics, University of Hamburg, Hamburg, Germany \\ Email: 0511814674-0001@t-online.de
}

How to cite this paper: Brink, H.-J. (2019) Do Near-Solar-System Supernovae Enhance Volcanic Activities on Earth and Neighbouring Planets on Their Paths through the Spiral Arms of the Milky Way, and What Might Be the Consequences for Estimations of Earth's History and Predictions for Its Future? International Journal of Geosciences, 10, 563-575.

https://doi.org/10.4236/ijg.2019.105032

Received: March 29, 2019

Accepted: May 12, 2019

Published: May 15, 2019

Copyright $\odot 2019$ by author(s) and Scientific Research Publishing Inc. This work is licensed under the Creative Commons Attribution International License (CC BY 4.0).

http://creativecommons.org/licenses/by/4.0/

\section{(c) (i) Open Access}

\begin{abstract}
Recent observations of young volcanism on the near-Earth terrestrial planets require a new understanding. Magmatic/volcanic episodes on Venus, Mars and Mercury, as well as on Earth's Moon, are apparently contemporaneous thermal events that accompanied increased magmatic/volcanic activity on Earth, following a 300-Myr cycle. A collateral galactic thermal source in the Milky Way appears to be needed that would predominantly affect the interior of the planets and, perhaps indirectly, Earth's biosphere, compared to other galactic sources, such as intense cosmic rays or large, rocky bolides. The search for such a source leads to near-Earth supernovae, with their neutrino output, and to the question of whether those neutrinos could act as energy transmitters to heat up the body of Earth, and also enhance its short-term magmatic processes; for example, Cenozoic anorogenic volcanism. This observation challenges present assumptions and paradigms about Earth's history, and requires the following reconsiderations: 1) the real origin of the end-Cretaceous mass extinction; 2) the general radioactive age determinations of rocks; and 3) geodynamic modelling using additional, external heat sources.
\end{abstract}

\section{Keywords}

Synchronous Planetary Magmatism, Galactic Heat Source,

Supernovae and White Dwarfs, Neutrinos, Mass Extinctions 


\section{Introduction}

Heat events in Earth's crust and mantle impact the melting of rocks, with the subsequent magmatism and volcanism enhancing the degassing of Earth's interior, influencing seafloor spreading and plate tectonics, governing the metamorphism of crustal rocks through successive volume reduction and water release [1] [2], and initiating the development of sedimentary basins with hydrocarbon generation therein. The heat flows in space and time (parabolic partial differential equation), which makes it difficult to define the exact attributes of distinct thermal anomalies. The causes and effects may overlap over a long time span, so that suspected changes in geological, biological and climatological processes do not happen in phase. Since heat transfer within Earth's body may be influenced by asteroid impacts, which may create shock-induced pathways for melting rocks through the crust and mantle [3], such sudden events may indicate at least a cosmic contribution apart from the intrinsic processes. However, starting with the global Phanerozoic magmatic cycle as an indication of 300-Myr-long periodic change in the Earth's heat flow, a further cosmic contribution to the thermal history of Earth can be suspected, since this periodicity has a galactic counterpart [4] [5].

Galactic cycles, caused by the path of the Solar System through the spiral arms of the Milky Way, and long-periodic geodynamic processes, both with durations of 150, 300 and $600 \mathrm{Myr}$, appear to be in phase. Due to cosmic ray fluctuations affecting cloud formation and the associated albedo of Earth, which has periodically led to glaciations every $150 \mathrm{Myr}$ (Figure 1), contemporaneous long-term climate changes are being caused. Sea-level fluctuates with glaciations, and the sizes of shallow seas vary synchronously. This causes periodic changes in the Moon's tidal dissipation, which presumably affects other geodynamic processes on Earth. The Moon may therefore directly or indirectly synchronise long-periodic Phanerozoic cycles (sea level, orogeny, magmatism, sedimentation, etc.) with the solar path through the spiral arms of the Milky Way. As sea-level fluctuations, orogeny, sedimentation and magmatism can be described as the pillars of an intrinsic geodynamic feedback system, no reasons appear to be required to allocate forces outside of the galactic-climatically-synchronised Earth-Moon system as the causes of these observed periodicities.

However, investigations into young volcanism on the near-Earth terrestrial planets require updated consideration. Increased magmatic/volcanic activities on Earth, following a 300-Myr cycle (Figure 2), have been accompanied by increased magmatic/volcanic episodes on Venus, Mars and Mercury, as well as on Earth's Moon [6]-[14], pointing to apparently synchronous planetary thermal events. Therefore, a common galactic thermal source within the spiral arms of the Milky Way appears to be required that would predominantly affect the interiors of these planets without having any recognisable direct effect on the life and geology on Earth. This excludes cosmic rays, due to their limited planetary-body penetration ability. Only neutrinos carry the ability to migrate deep into, 
Spiral arm passageways and glacial epochs

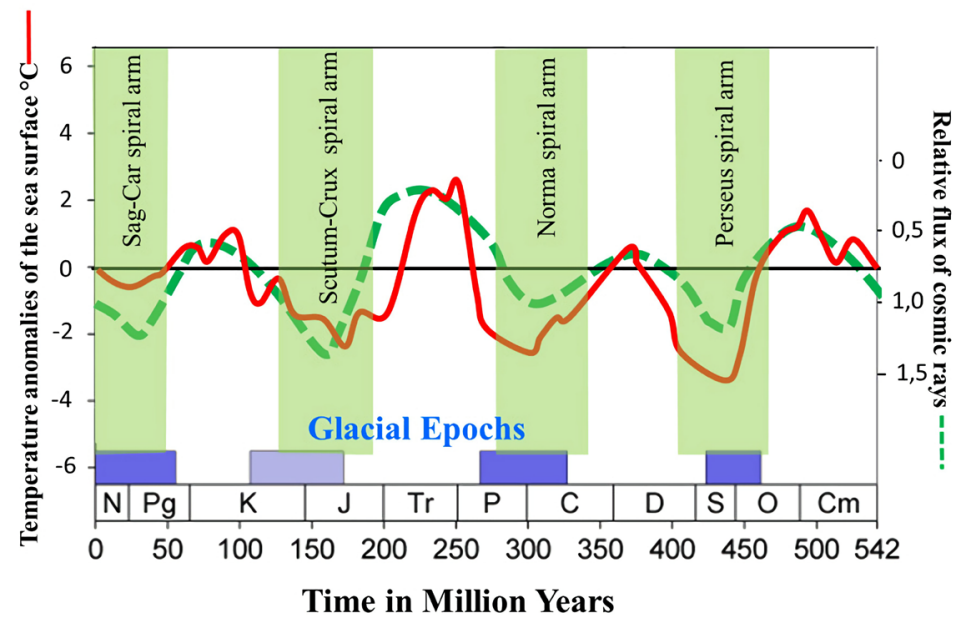

Figure 1. Phanerozoic cycles ( $150 \mathrm{Myr}$ ) of tropical sea-surface temperatures (red line), shown against greenhouse and glacial epochs and the flux of cosmic radiation (dashed green line, scale inverted), which correlate with the passage of Earth through the spiral arms of the Milky Way. The resulting increase in cosmic ray flux enhanced cloud formation by the generation of condensation nuclei. This led to a temperature decrease. Iron meteorites are regarded to be a medium for the cosmic ray flux signal [5]. Source: [4].

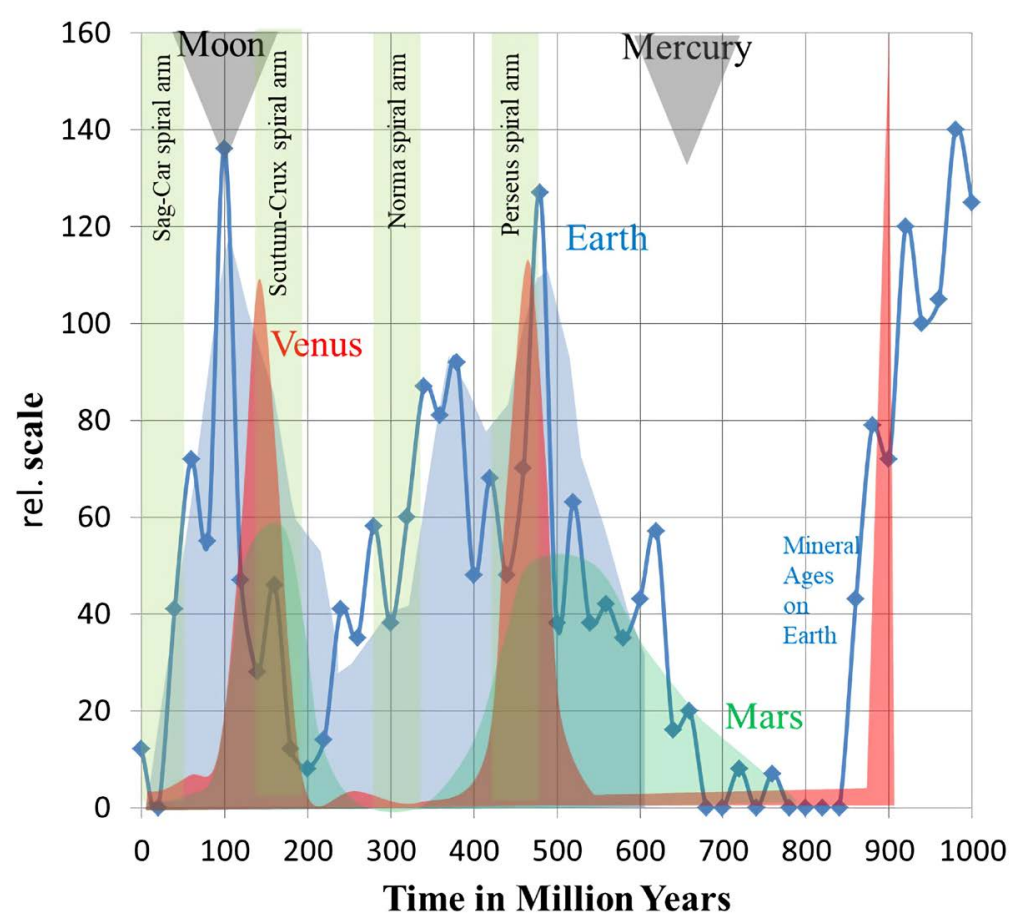

Figure 2. Compilation of magmatic/volcanic (heating) events on Earth [6] [7], Mars [8] [9] [10] (sketchy model of the Martian chronology) and Venus [11] (adjusted to absolute time) during the Phanerozoic and Neoproterozoic (about the last $1000 \mathrm{Myr}$ ). These planetary events appear to have been contemporaneous episodes that were accompanied by similar, young magmatic activities on Mercury [12] [13] (possibly about $650 \mathrm{Ma}$ ) and on Earth's moon [14]) (possibly about $100 \mathrm{Ma}$ ), pointing to an extraplanetary (galactic) cause. This may have been related to the passage of the Solar System through the denser spiral arms Perseus and Scutum-Crux of the Milky Way, with a 300 Myr cycle. Source: [4]. 
or through, Earth. Dark matter and dark energy, as optional sources, may gravitationally affect the rotation of stars within galaxies or properties of the expanding universe, but may not be able to govern millions-of-years-long periodic changes in the relatively "tiny" Solar System. Supernova explosions in the spiral arms, the preferred birth and death place for stars in the Milky Way, are one main source of neutrinos, which carry about $98 \%$ of the emitted, released gravitational energy. The rest are cosmic rays. Many of the supernovae convert to so-called white dwarfs, whose cooling age and distance from Earth can be convincingly estimated. Larger stars convert into neutron stars or black holes. One large star of interest is certainly the roughly 8 - 9-Myr-young supergiant Betelgeuse (which has never left its spiral arm!) in the Orion constellation, at a distance of about 600 light years (184 parsecs [pc]), with a mass about 1000 times larger than our Sun (which has an age of $4600 \mathrm{Myr}$, and has already performed seven to eight orbits around the rotating Milky Way and passed the four spiral arms in total about 30 times) and a death expected between now and 100,000 years hence. The distribution of near-Solar-System white dwarfs [15] accompanying the Solar System, with an assumed similar rotation speed around the galactic centre, indicates a $150-\mathrm{Myr}$ periodicity of maxima along the time axis of solar passages through the spiral arms of the Milky Way (Figure 3). Properties

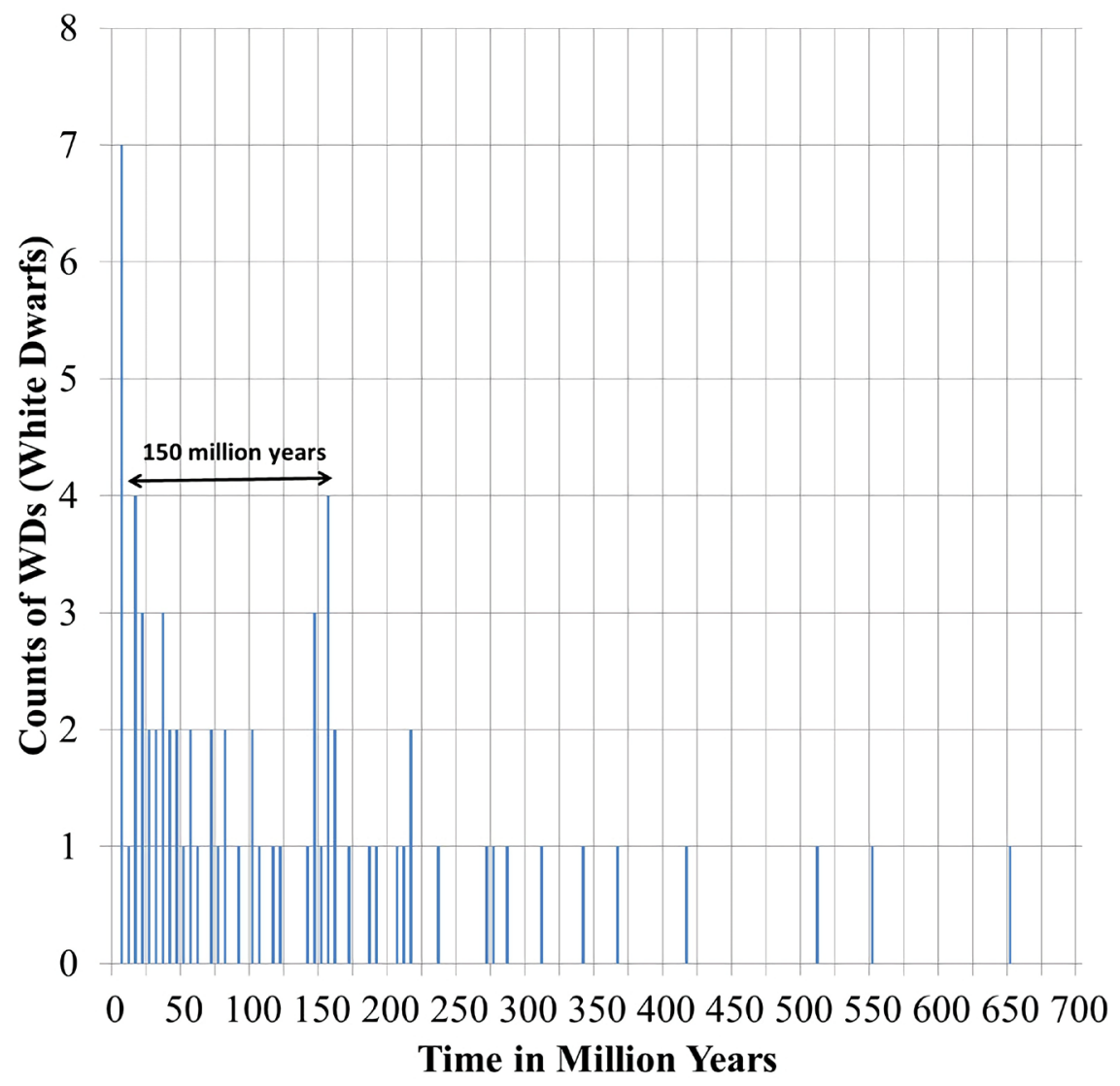

Figure 3. Counts of near-Earth white dwarfs per steps of 5 Myr, after [15], showing a 150 Myr periodicity (one repetition recognisable). 
of the spiral arms may therefore synchronise white dwarf development and enhance the intermediate status of stars in their supernova stage, or the time span between birth and death for many of these stars should be shorter than $75 \mathrm{Myr}$ (estimated residence time in a spiral arm). Additional supporting data can be found in [16] [17] [18]. However, short-lived stars are most likely less probable in the Milky Way than normal stars like the Sun. Probably due to their limited detectability, the number of older white dwarfs significantly decreases on the timeline. Therefore the suspected 300-Myr cycle remains yet undiscovered using this dataset. The importance of the spiral arms, especially their high-density regions, on passing stars has been supported by [19], who investigated methanol masers as arm tracers. They revealed that asteroid/comet impacts on Earth are considerably grouped close to spiral arms and within specific locations of an average arm structure. The Permian-Triassic and Cretaceous-Paleogene boundary extinctions occurred during solar passages through small star-formation regions in two different arms. The origin of the Solar System occurred in a similar region in a third spiral arm.

\section{Planetary Magmatism}

If the 300-Myr cycle of magmatism on Earth refers to a galactic cause, or is at least influenced by the properties and processes of the Milky Way (e.g. two out of four denser spiral arms), can episodic volcanic events on Earth perhaps tell us something about a cosmic contribution? Do heat events, needed for the melting of rocks, correlate with cosmic incidents, especially with supernova explosions and their freeing of neutrinos? A correlation of episodes of hotspot volcanism as global pulses during the last $70 \mathrm{Myr}$ [20], including episodes of large igneous provinces and the ages of near-Earth white dwarfs [15], suggests a high coincidence (Figure 4). The majority of their maxima times correlate significantly with each other. Since the error in white dwarf ages amounts to an average of roughly $20 \%$, the real coincidences may be even better. Furthermore, the diffluence of heat events may have also contributed to some uncertainties. On a regional scale, a prolific example, with a remarkable coincidence of magmatic pulses [20] with supernovae explosions [15], is the intense (Cenozoic) anorogenic volcanism in Germany during the last 70 Myr (Figure 5). The past magma chambers in its subsurface, within or below the Variscan crust, seem to have been highly sensitive to the suspected cosmic causes. It has indeed been demonstrated that the complex properties of the central European Variscan crust have caused specific geological reactions regionally, as well as locally [2] [21]. A study of the enhancement of already active or sleeping magmatism as a cause for global pulses of hotspot volcanoes and mantle plumes may lead to an assumption of a causal relationship with cosmic heating events that should then also be generally valid for the 300-My periodicity of global magmatism and related seafloor spreading rates. The contribution of supernovae and the physical ability of their neutrinos are therefore interesting issues for further investigation. 


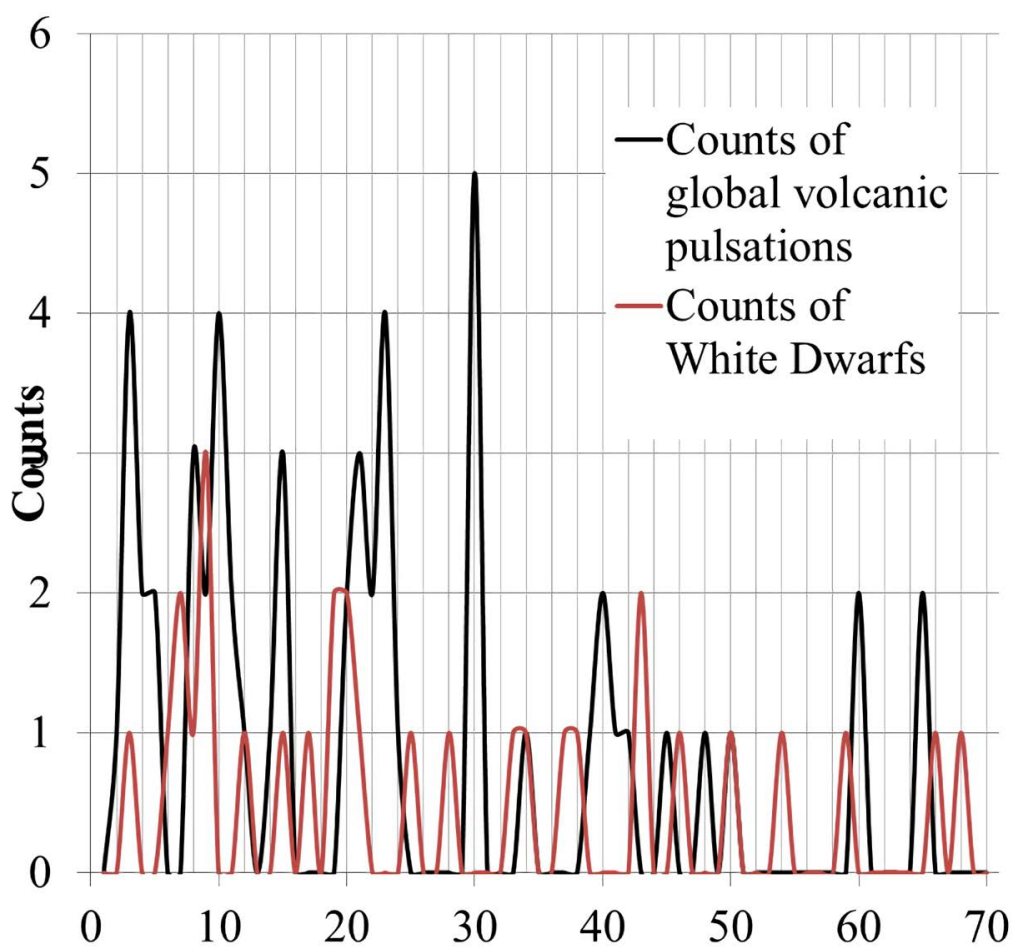

Figure 4. Global pulses of hotspot volcanism [20] and near-Earth white dwarfs, after [15]. The first white dwarf count at about $3 \mathrm{Ma}$ is related to a supernova, as identified by [28].

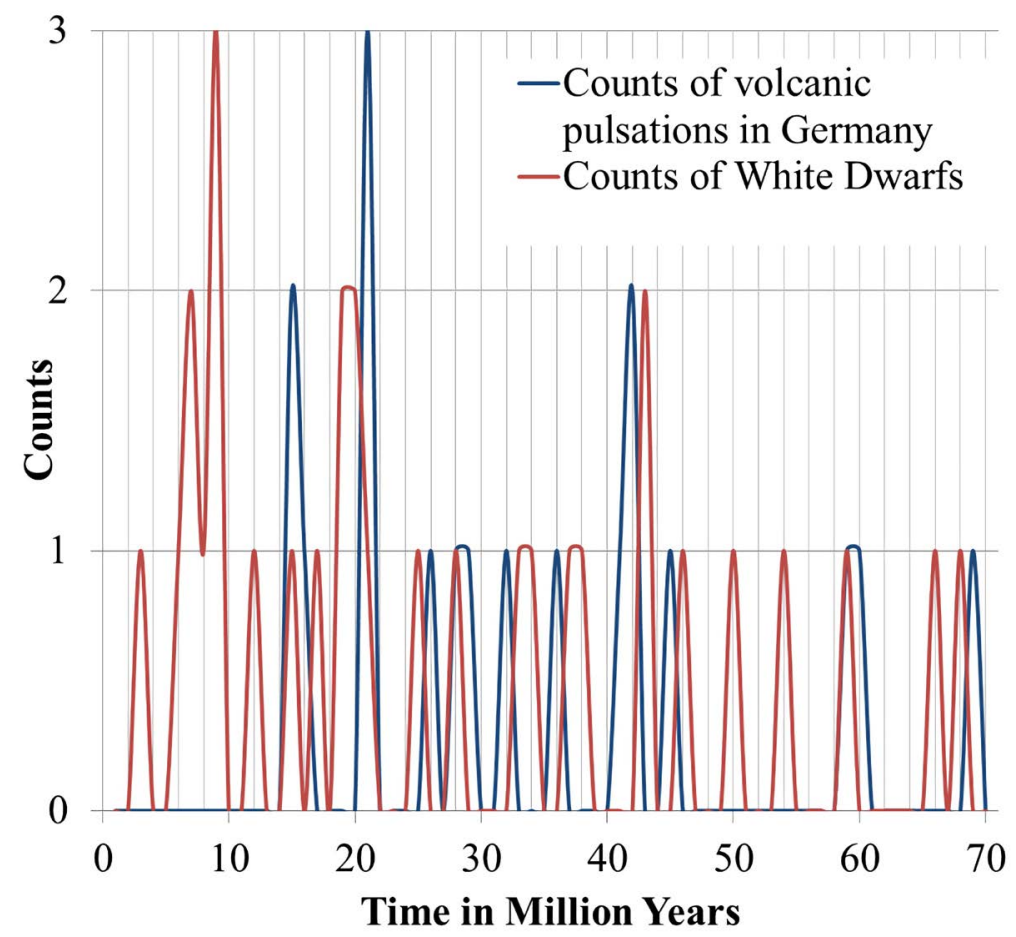

Figure 5. Volcanic pulses in Germany during the last $70 \mathrm{Myr}$, after [20], and near-Earth white dwarfs, after [15].

Even if the optical correlation between the two datasets in Figure 5 appears to be significantly similar to those in Figure 1 (after [5]) and Figure 2 (after [4]), a 
mathematical analysis, by applying a cross-correlation, $R_{x y}[n]$, to these two discrete functions (such as $x$ versus $y$ ), could help to prove and correct their possible causal relationships. With $m=$ series and $n=$ time shift, in 1-Myr steps, and with:

$$
R_{x y}[n]=\sum x[m] * y[m+n], m \text { from }-\infty \text { to }+\infty,
$$

an average optimal time delay of only +2 Myr appears appropriate. With this time shift, which adds 2 Myr to the cooling age of the investigated white dwarfs, the cross-correlation function shows a maximal relative value of 45 , compared to 38 and 36 for delays of $+1 \mathrm{Myr}$ and $+3 \mathrm{Myr}$, respectively. The analysed delay remains well within the tolerance limits of both datasets, also keeping in mind that heat flows in space and time, and that a cosmic heat cause should precede volcanism on Earth as apossible effect.

\section{Neutrinos}

Neutrinos are currently being intensively discussed in the scientific community (e.g. [22]-[27]). The bulk of (un)published papers is witness to this activity, and demonstrates the presence of many unanswered questions. For the purpose of this paper, the possible ability of neutrinos to transfer energy to matter within Earth, and the subsequent generation of heat thereby, is the only important issue, in a general sense. All other findings will only be mentioned where necessary.

The wide range of neutrino interactions encompasses coherent scattering, neutrino capture, inverse beta decay, low-energy nuclear interactions, quasi-elastic scattering, resonant pion production, kaon production, deep inelastic scattering and ultra-high-energy interactions [22]. Among these, the low-energy interactions may be the relevant interactions for both supernova and solar neutrinos. Important elements in Earth's crust, mantle and core play a decisive role on the matter side, as counterparts for the approaching low-energy neutrinos, namely, iron, nickel and the radioactive isotopes of uranium, thorium and potassium. In the core, the isotopes ${ }^{57} \mathrm{Fe}$ and ${ }^{61} \mathrm{Ni}$ can harvest low energies of $14 \mathrm{keV}$ and $67 \mathrm{keV}$, respectively, from concentrated neutrino currents, and then convert this to heat [23]. According to [24], ${ }^{56} \mathrm{Fe}$ and ${ }^{208} \mathrm{~Pb}$ isotopes may have the ability to capture supernova neutrinos, and can excite the nuclei of the isotopes beyond their neutron-emission thresholds through charged-current and neutral-current interactions. Isotopes of other frequent elements in Earth's crust, mantle and core may interact with neutrinos in similar ways. Emitted neutrons can be used as a signal for a supernova event. However, interacting neutrons may enhance the radioactive decay of uranium, thorium and potassium (e.g. in Earth's crust and mantle), should neutrinos themselves not be able to interact with the nuclei accordingly, and free the stored energy by converting it into heat. In terms of heating up Earth's interior, the question remains as to whether such reactions occur frequently enough; the supernova neutrino flux must repeatedly exceed the general cosmic background flux by a very significant amount. The suggested 
enhancement of the radioactive decay rate significantly challenges the paradigm that the natural decay rates of radioactive isotopes are constant. In case this paradigm has to be reconsidered, the current radioactive age determinations of rocks must be reinvestigated.

\section{Supernovae}

To guarantee a high flux of neutrinos during the passage of the Solar System through the spiral arms of the Milky Way, a high rate of supernova explosions close to Earth is required. According to [28], every 2 - 4 Myr, a supernova explodes in Earth's local galactic neighbourhood (still in the Orion spur of the Sagittarius-Carina spiral arm), within a distance of about 100 pc (326 light-years). Radionuclides of the interstellar dust, with half-lives of up to $100 \mathrm{Myr}$, may be signals of quite recent massive-star and supernova activity close to Earth. One such radionuclide is ${ }^{60} \mathrm{Fe}$ (with a half-life of $2.6 \mathrm{Myr}$ ), which is ejected in supernova explosions and winds from massive stars. ${ }^{60} \mathrm{Fe}$ signals of interstellar origin from multiple events, recorded in crusts of deep-sea nodules, are globally distributed over a longer period. Results suggest that ${ }^{60} \mathrm{Fe}$ interstellar influxes onto Earth occurred at $1.5-3.2 \mathrm{Ma}$ and at $6.5-8.7 \mathrm{Ma}$.

The second of these exploded at the time Betelgeuse was born. For an estimated period of $75 \mathrm{Myr}$, during the passage of the Solar System through the current spiral arm, between 20 and 40 supernovae may have exploded close to Earth. This is in line with the observation of [15], who counted 30 white dwarfs, as remnants of supernovae, within a distance of $70 \mathrm{pc}$ (228 light-years). Neutron stars and black holes have not been incorporated, but their progenitor supernovae may have contributed to the neutrino flux as well, despite their probable low number. The energy released by a supernova via neutrinos amounts to $2-3 \times$ $10^{53}$ erg or $2-3 \times 10^{43} \mathrm{~kJ}$, with 30 supernovae between 6 and $9 \times 10^{44} \mathrm{~kJ}$ occurring through a 75-Myr time span. To heat up the entire Earth by an estimated $10^{\circ} \mathrm{C}$, about $1.2 \times 10^{25} \mathrm{~kJ}$ would be required $(\Delta Q$ (heat) $=c \times m \times \Delta T$ with $\mathrm{m}$ (mass of Earth $)=6 \times 10^{24} \mathrm{~kg}$ and $c$ (specific heat $\left.)=2 \mathrm{~kJ} / \mathrm{kg}^{\circ} \mathrm{C}\right)$. The distances and directions between the supernovae and the Solar System, how the neutrinos spread geometrically (importance of the applied exponent of distance; dependence of star rotation?), how long the paths of the planets last in the neutrino flux are, as well as the properties of the matter of the terrestrial solar planets and their interactions with the emitted neutrinos, are the limiting factors that control the suspected heating of these planetary bodies. A temperature increase of $10^{\circ} \mathrm{C}$ may be enough to enhance the melting of larger rock volumes that are already in an intermediate stage between solid and melt.

\section{Mass Extinctions}

Should the enhancement of volcanic activity on Earth be governed by MilkyWay-related cosmic heating sources (presumably supernovae), then there are further topics that will come under intense scientific scrutiny, such as the time- 
line of mass extinction intensities, volcanism and asteroid impacts [29]. In addition, the results of [30] suggested that the hypothesis of periodic impacts and extinction events is still viable, even though a periodicity of about $26-27 \mathrm{Myr}$ appears to be dominant. In particular, the asteroid impact in present-day Yuca$\tan /$ Mexico that currently defines the intense end-Cretaceous mass extinction may be worth reinvestigation. This event was attended by intense volcanic eruptions in India (the Deccan Traps), which bears similarity to the eruption of the Siberian Traps in Russia at the dramatic end-Permian mass extinction. Based on the iridium anomaly found in globally-distributed sediments, and the missing plutonium-244 $\left({ }^{244} \mathrm{Pu}\right)$ signal, previously required as an accompanying signature to a supernova explosion [31], an impacting cosmic bolide appeared to be the only possible cause of the end-Cretaceous mass extinction. However, recent investigations into the distribution of ${ }^{244} \mathrm{Pu}$ in space and on Earth have indicated that a general plutonium signal is no longer required for a supernova [32]. Therefore, a supernova can still be a possible cause of the end-Cretaceous mass extinction. Extinctions during the last $10 \mathrm{Myr}$ and before have already been correlated with supernovae (e.g. [33] [34]). That asteroid impacts may have been caused by supernovae debris pushed in the direction of the Solar System, or through the stability of the asteroid belts of the Solar System being affected by galactic forces, has been discussed intensively by [35], who cited many controversial papers. Near-Earth supernova explosions, thermally-delayed volcanism, accompanying asteroid impacts and mass extinctions, possibly caused or enhanced by the attendant supernova cosmic rays, can be linked together on a smeared timeline. As a number of asteroid impacts predate hotspot volcanism at plate-tectonically-corrected identical locations by about 10 to $20 \mathrm{Myr}$ [3], certainly not every impact and volcanic eruption may belong to a supernova-controlled system, but cause and effect between the aforementioned four fundamental activities during the evolution of Earth appear to be tentatively related to each other. The Universe, with our Milky Way stars, is the master and the geodynamic processes on Earth represent the slaves, in a system-theoretical view. Within Earth, a subsequent master-slave system of geodynamic cycles [1] has been established as a follow-up at least since Phanerozoic times. The ruling forces coming from space do not support the Gaia hypothesis [36] that Earth and the life on it are effectively controlling their own destiny through intrinsic processes.

\section{Conclusion}

Based on the above, it can be concluded that a steady cosmic contribution to the geological evolution of Earth appears obvious, and that the coincidence of near-Earth supernovae with magmatic events and mass extinctions is remarkably high. Should it be supported by other researchers, this observation challenges present assumptions and paradigms about Earth's history and predictable future, and requires the following reconsiderations: 1) the real origin of the end-Cretaceous mass extinction; 2) the general radioactive age determinations of rocks; and 3) 
geodynamic modelling using additional, external heat sources. Should the standard knowledge on neutrinos-that they interact very weakly with matter and no exceptions are possible-be an unsurmountable barrier, then other, as yet unknown, cosmic forces that are able to synchronously heat planets in the Solar System must be identified.

\section{Acknowledgements}

The author would like to thank Aaron Toussaint for providing help with the language, and all those colleagues who supported these ideas. Many thanks go also to the anonymous reviewers, whose recommendations and suggested corrections and additions to improve the paper were deeply appreciated.

\section{Conflicts of Interest}

The author declares no conflicts of interest regarding the publication of this paper.

\section{References}

[1] Brink, H.-J. (2006) Do the Global Geodynamic Cycles of the Phanerozoic Represent a Feedback System of the Earth and Is the Moon Involved as an Acting External Force? Zeitschrift der Deutschen Gesellschaft für Geowissenschaften-German Journal of Geology, 157, 17-40. https://doi.org/10.1127/1860-1804/2006/0157-0017

[2] Brink, H.-J. (2009) Mantle Plumes and the Metamorphism of the Lower Crust and Their Influence on Basin Evolution. Marine and Petroleum Geology, 26, 606-614. https://doi.org/10.1016/j.marpetgeo.2009.02.002

[3] Brink, H.-J. (2012) Flood Basalts, Mantle Plumes and Asteroid Impacts. In: West, J.P., Ed., Basalt. Types, Petrology and Uses, Nova Science Publishers, Hauppauge, 1-32.

[4] Brink, H.J. (2015) Periodic Signals of the Milky Way Concealed in Terrestrial Sedimentary Basin Fills and in Planetary Magmatism? International Journal of Geosciences, 6, 831-845. https://doi.org/10.4236/ijg.2015.68067

[5] Shaviv, N.J. and Veizer, J. (2003) Celestial Driver of Phanerozoic Climate? GSA Today, 13, 4-10. https://doi.org/10.1130/1052-5173(2003)013<0004:CDOPC>2.0.CO;2

[6] Engel, A.E.J. and Engel, C.G. (1964) Continental Accretion and the Evolution of North America. In: Subramaniam, A.P. and Balakrishna, S., Eds., Advancing Frontiers in Geology and Geophysics, Indian Geophysical Union, Hyderabad, 17-37.

[7] Gastil, G. (1960) The Distribution of Mineral Dates in Time and Space. American Journal of Science, 258, 1-35. https://doi.org/10.2475/ajs.258.1.1

[8] Hartmann, W.K. and Neukum, G. (2001) Cratering Chronology and the Evolution of Mars. Space Science Reviews, 96, 165-194. https://doi.org/10.1023/A:1011945222010

[9] Werner, S.C. (2009) The Global Martian Volcanic Evolutionary History. Icarus, 20, 44-68. https://doi.org/10.1016/j.icarus.2008.12.019

[10] Robbins, S.J., Di Achille, G. and Hynek, B.M. (2011) The Volcanic History of Mars: High-Resolution Crater-Based Studies of the Calderas of 20 Volcanoes. Icarus, 211, 1179-1203. https://doi.org/10.1016/j.icarus.2010.11.012 
[11] Basilevsky, A.T. and Head, J.W. (2002) Venus: Analysis of the Degree of Impact Crater Deposit Degradation and Assessment of Its Use for Dating Geological Units and Features. Journal of Geophysical Research, 107, 5-1-5-38. https://doi.org/10.1029/2001JE001584

[12] Thomas, R.J., Rothery, D.A., Conway, S.J. and Anand, M. (2014) Long-Lived Explosive Volcanism on Mercury. Geophysical Research Letters, 41, 6084-6092. https://doi.org/10.1002/2014GL061224

[13] Thomas, R.J., Rothery, D.A., Conway, S.J. and Anand, M. (2015) The Timing and Distribution of Pyroclastic Volcanism on Mercury. The Open University, Milton Keynes.

[14] Braden, S.E., Stopar, J.D., Robinson, M.S., Lawrence, S.J., van der Bogert, C.H. and Hiesinger, H. (2014) Evidence for Basaltic Volcanism on the Moon within the Past 100 Million Years. Nature Geoscience, 7, 787-791. https://doi.org/10.1038/ngeo2252

[15] Zhao, J.K., Luo, A.L., Oswalt, T.D. and Zhao, G. (2013) 70 DA White Dwarfs Identified in Lamost Pilot Study. The Astronomical Journal, 145, 169. https://doi.org/10.1088/0004-6256/145/6/169

[16] Sion, E.M., Holberg, J.B., Oswalt, T.D., McCook, G.P., Wasatonic, R. and Myszka, J. (2014) The White Dwarfs within 25 pc of the Sun: Kinematics and Spectroscopic Subtypes. The Astronomical Journal, 147, 129. https://doi.org/10.1088/0004-6256/147/6/129

[17] Tremblay, P.-E., Kalirai, J.S., Soderblom, D.R., Cignoni, M. and Cummings, J. (2014) White Dwarf Cosmochronology in the Solar Neighborhood. The Astrophysical Journal, 791, Article ID: 92. https://doi.org/10.1088/0004-637X/791/2/92

[18] Torres, S. and García-Berro, E. (2016) The White Dwarf Population within 40 pc of the Sun. $A \& A, 588$, A35. https://doi.org/10.1051/0004-6361/201528059

[19] Gillman, M.P., Erenler, H.E. and Sutton, P.J. (2018) Mapping the Location of Terrestrial Impacts and Extinctions onto the Spiral Arm Structure of the Milky Way. International Journal of Astrobiology, 10, 1-6. https://doi.org/10.1017/S1473550418000125

[20] Mjelde, R., Wessel, P. and Müller, R.D. (2010) Global Pulsations of Intraplate Magmatism through the Cenozoic. Geological Society of America, Lithosphere, 2, 361-376. https://doi.org/10.1130/L107.1

[21] Brink, H.-J. (2011) The Crustal Structure around the Harz Mountains (Germany): Review and Analysis. Zeitschrift der Deutschen Gesellschaft für Geowissenschaften, 162, 235-250. https://doi.org/10.1127/1860-1804/2011/0162-0235

[22] Formaggio, J.A. (2013) From eV to EeV: Neutrino Cross-Sections across Energy Scales.

[23] Wei, Y. (2017) Converged Solar Neutrinos Heat Outer Core of Earth to Liquid. Working Paper, Kiwaho Laboratory of Energy and Ecology Inc., Ontario. https://www.researchgate.net/publication/316602230

[24] Paar, N., Suzuki, T, Honma, M., Marketin, T. and Vretenar, D. (2011) Uncertainties in Modeling Low-Energy Neutrino-Induced Reactions on Iron-Group Nuclei. Physical Review C, 84, Article ID: 047305. https://doi.org/10.1103/PhysRevC.84.047305

[25] Renshaw, A., Abe, K., Hayato, Y., Iyogi, K., Kameda, J., Kishimoto, Y., Miura, M., Moriyama, S., Nakahata, M., Nakano, Y., Nakayama, S., Sekiya, H., Shiozawa, M., Suzuki, Y., Takeda, A., Takenaga, Y., Tomura, T., Ueno, K., Yokozawa, T., Wendell, R.A., Irvine, T., Kajita, T., Kaneyuki, K., Lee, K.P., Nishimura, Y., Okumura, K., 
McLachlan, T., Labarga, L., Berkman, S., Tanaka, H.A., Tobayama, S., Kearns, E., Raaf, J.L., Stone, J.L., Sulak, L.R., Goldhabar, M., Bays, K., Carminati, G., Kropp, W.R., Mine, S., Smy, M.B., Sobel, H.W., Ganezer, K.S., Hill, J., Keig, W.E., Hong, N., Kim, J.Y., Lim, I.T., Akiri, T., Himmel, A., Scholberg, K., Walter, C.W., Wongjirad, T., Ishizuka, T., Tasaka, S., Jang, J.S., Learned, J.G., Matsuno, S., Smith, S.N., Hasegawa, T., Ishida, T., Ishii, T., Kobayashi, T., Nakadaira, T., Nakamura, K., Oyama, Y., Sakashita, K., Sekiguchi, T., Tsukamoto, T., Suzuki, A.T., Takeuchi, Y., Bronner, C., Hirota, S., Huang, K., Ieki, K., Ikeda, M., Kikawa, T., Minamino, A., Nakaya, T., Suzuki, K., Takahashi, S., Fukuda, Y., Choi, K., Itow, Y., Mitsuka, G., Mijakowski, P., Hignight, J., Imber, J., Jung, C.K., Yanagisawa, C., Ishino, H., Kibayashi, A., Koshio, Y., Mori, T., Sakuda, M., Yano, T., Kuno, Y., Tacik, R., Kim, S.B., Okazawa, H., Choi, Y., Nishijima, K., Koshiba, M., Totsuka, Y., Yokoyama, M., Martens, K., Marti, Ll., Vagins, M.R., Martin, J.F., de Perio, P., Konaka, A., Wilking, M.J., Chen, S., Zhang, Y. and Wilkes, R.J. (2014) First Indication of Terrestrial Matter Effects on Solar Neutrino Oscillation. Physical Review Letters, 112, Article ID: 091805. https://doi.org/10.1103/PhysRevLett.112.091805

[26] Khavroshkin, O.B. and Tsyplakov, V.V. (2016) The Radioactivity of Nuclei \& Solar Oscillations: New Experiments. Natural Science, 8, 20-32.

https://doi.org/10.4236/ns.2016.81003

[27] Bandyopadhyay, A., Bhattacharjee, P., Chakraborty, S., Kar, K. and Saha, S. (2017) Detecting Supernova Neutrinos with Iron and Lead Detectors. Physical Review D, 95, Article ID: 065022. https://doi.org/10.1103/PhysRevD.95.065022

[28] Wallner, A., Feige, J., Kinoshita, N., Paul, M., Fifield, L.K., Golser, R., Honda, M., Linnemann, U., Matsuzaki, H., Merche, S., Rugel, G., Tims, S.G., Steier, P., Yamagata, T. and Winkler, S.R. (2016) Recent Near-Earth Supernovae Probed by Global Deposition of Interstellar Radioactive ${ }^{60} \mathrm{Fe}$. Nature, 532, 69-72.

https://doi.org/10.1038/nature17196

[29] White, R.V. and Saunders, A.D. (2005) Volcanism, Impact and Mass Extinctions: Incredible or Credible Coincidences? Lithos, 79, 299-316. https://doi.org/10.1016/j.lithos.2004.09.016

[30] Rampino, M.R. and Caldeira, K. (2015) Periodic Impact Cratering and Extinction Events over the Last 260 Million Years. Monthly Notices of the Royal Astronomical Society, 454, 3480-3484. https://doi.org/10.1093/mnras/stv2088

[31] Alvarez, L.W., Alvarez, W., Asaro, F. and Michel, H.V. (1980) Extraterrestrial Cause for the Cretaceous-Tertiary Extinction. Science, 208, 1095-1108.

https://doi.org/10.1126/science.208.4448.1095

[32] Wallner, A., Faestermann, T., Feige, J., Feldstein, C., Knie, K., Korschinek, G., Kutschera, W., Ofan, A., Paul, M., Quinto, F., Rugel, G. and Steier, P. (2015) Abundance of Live $244 \mathrm{Pu}$ in Deep-Sea Reservoirs on Earth Points to Rarity of Actinide Nucleosynthesis. Nature Communications, 6, Article No. 5956. https://doi.org/10.1038/ncomms6956

[33] Svensmark, H. (2012) Evidence of Nearby Supernovae Affecting Life on Earth. Monthly Notices of the Royal Astronomical Society, 423, 1234-1253. https://doi.org/10.1111/j.1365-2966.2012.20953.x

[34] Korschinek, G. (2016) Mass Extinctions and Supernova Explosions. In: Murdin, P. and Alsabeti, A., Eds., Handbook of Supernovae, Springer International Publishing, Berlin, 1-12. https://arxiv.org/ftp/arxiv/papers/1609/1609.02817.pdf https://doi.org/10.1007/978-3-319-20794-0_22-1

[35] LaViolette, P.A. (2009) The Cause of the Megafaunal Extinction: Supernova or Ga- 
lactic Core Outburst? (Twenty-Two Problems with the Firestone-West Supernova Comet Theory). The Starburst Foundation, Schenectady.

http://starburstfound.org/YDextinct/p1.html

[36] Lovelock, J. (2000) Gaia: A New Look at Life on Earth. 3rd Edition, Oxford University Press, Oxford. 